\title{
MANEJO DE LA FERTILIZACIÓN FOSFORADA EN EL CULTIVO DE MAÍZ, EL EJIDO, PANAMÁ 2004-2013 ${ }^{1}$
}

\author{
Roman Gordon-Mendoza², Jorge Enrique Franco-Barrera², José Ezequiel Villarreal-Núñez ${ }^{2}$, T. Jot Smith ${ }^{3}$
}

\section{RESUMEN}

Manejo de la fertilización fosforada en el cultivo de maíz, El Ejido, Panamá 2004-2013. El objetivo del presente trabajo fue determinar, en el cultivo de maíz, el efecto del manejo de la fertilización fosforada a mediano y largo plazo con dos métodos de aplicación. Se estableció un experimento en El Ejido, Panamá, del 2004 al 2013 en un suelo Udic Haplustalf. Se evaluaron dos componentes en un factorial $5 \times 4$ con arreglo de fajas bajo un diseño de bloques al azar con tres repeticiones. En la parcela principal se aplicó $\mathrm{P}$ al voleo en dosis de 0, 25, 50, 75 y $100 \mathrm{kgP} / \mathrm{ha}$ (aplicado sólo el primer año), en las sub-parcelas se aplicó durante diez años, $\mathrm{P}$ en banda junto al $\mathrm{N}$ y $\mathrm{K}$ en dosis de $0,10,20$ y $30 \mathrm{kgP} / \mathrm{ha}$. El máximo rendimiento de grano se logró con la aplicación acumulada de $304 \mathrm{~kg}$ (en promedio $30,4 \mathrm{kgP} / \mathrm{año}$ ). El efecto residual de las aplicaciones de $0 \mathrm{y}$ $25 \mathrm{kgP} / \mathrm{ha}$ al voleo presentaron un rendimiento relativo por debajo del $80 \%$. El tratamiento de $50 \mathrm{kgP} /$ ha se mantuvo en 82\%, mientras que el de 75 disminuyó a partir del año 2007 a un valor cercano al $80 \%$. El tratamiento de $100 \mathrm{kgP} / \mathrm{ha}$ al voleo presentó un rendimiento relativo cercano al $100 \%$. La aplicación al voleo de más de $50 \mathrm{kgP} /$ ha no difirió de la respuesta obtenida con aplicaciones anuales de $30 \mathrm{P} / \mathrm{ha}$ en los primeros cinco años. Estos resultados sugieren que el efecto residual de la aplicación del fósforo al voleo e incorporado previo a la siembra, bajo el tipo de suelo empleado varió según la dosis aplicada inicialmente, en donde las dosis superiores a $50 \mathrm{~kg} / \mathrm{ha}$ deben repetirse después de seis años para mantener una buena productividad, ya que, a partir de este tiempo el efecto residual se redujo en forma drástica.

Palabras claves: voleo, efecto residual, nivel crítico, óptimo físico.

\begin{abstract}
Phosphorus fertilization management in corn crop, EI Ejido, Panama 2004-2013. The aim of this study was to determine medium and long management of phosphorus fertilization with two application methods on the maize. An experiment was established in El Ejido, Panama, from 2004 to 2013 on Udic Haplustalf soil. Two factors were evaluated in a $5 \times 4$ factorial arrangement of split plot under a randomized block design with three replications. The main plot was the broadcast application of $\mathrm{P}$ in rates of $0,25,50$, 75 , and $100 \mathrm{kgP} / \mathrm{ha}$ (applied only the first year), while in subplots applied $\mathrm{P}$ in band, for ten years, together with $\mathrm{N}$ and $\mathrm{K}$ doses of $0,10,20$ and $30 \mathrm{kgP} / \mathrm{ha}$. The maximum grain yield was achieved by applying average $304 \mathrm{~kg}$ (average of 30.4 $\mathrm{kgP} /$ year). It was observed that the residual effect broadcast applications of 0 and $25 \mathrm{kgP} / \mathrm{ha}$ had a relative yield below $80 \%$. Treatment of $50 \mathrm{kgP} / \mathrm{ha}$ remained at $82 \%$, while 75 $\mathrm{kgP} / \mathrm{ha}$ declined from the year 2007 and is at a value close to $80 \%$. Treatment of $100 \mathrm{kgP} / \mathrm{ha}$ broadcast presents a relative yield close to $100 \%$. The results indicate that the broadcast application over $50 \mathrm{kgP} / \mathrm{ha}$ did not differ from the response obtained with annual applications of $30 \mathrm{P} / \mathrm{ha}$ in the first five years. These results suggest that the residual effect of phosphorus broadcast and incorporated before planting, on the type of soil used, varies according doses applied initially, where dosages above $50 \mathrm{~kg} / \mathrm{ha}$ should be repeated after six years to maintain good productivity, since, from this time the residual effect is drastically reduced.
\end{abstract}

Keywords: broadcast, residual effect, critical level, optimal physical.

Recibido: 30 abril, 2015. Aceptado: 28 de julio, 2015. Investigación financiada por el proyecto Manejo integral del cultivo de maíz del Instituto de Investigación Agropecuaria de Panamá (IDIAP), Panamá.

2 Instituto de Investigación Agropecuaria de Panamá (IDIAP), Panamá.gordon.roman@gmail.com, joenfra13@gmail.com, jevilla38@gmail.com

3 North Carolina State University PO Box 7619, Raleigh, North Carolina 27695-7619, NCSU, USA. jot_smith@ncsu.edu

(c) (1) $\Theta 2016$ Agronomía Mesoamericana es desarrollada en la Universidad de Costa Rica y se encuentra licenciada con Creative Commons Reconocimiento-NoComercial-SinObraDerivada 3.0 Costa Rica. Para más información escríbanos a pccmca@ucr.ac.cr 


\section{INTRODUCCIÓN}

El fósforo (P) es después del nitrógeno, el segundo elemento más importante para el crecimiento de las plantas, la producción de los cultivos y su calidad; además, es uno de los nutrimentos que más limita la producción agrícola en los trópicos. La agricultura moderna depende del $\mathrm{P}$ derivado de roca fosfórica, la cual es un recurso no renovable y cuyas reservas mundiales actuales podrían agotarse en 50-100 años (Cordell et al., 2009; Dawson y Hilton, 2011), por lo que aumentar aún más el uso de fertilizantes fosforados, en el contexto de una creciente demanda mundial de productos agrícolas, no parece una opción viable (Cordell et al., 2009; Khan y Hanjra, 2009; Hinsinger et al., 2011). La planta absorbe la mayor cantidad de $\mathrm{P}$ como ión ortofosfato primario $\left(\mathrm{H}_{2} \mathrm{PO}^{-4}\right)$, seguido del ión ortofosfato secundario $\left(\mathrm{H}_{2} \mathrm{PO}^{=4}\right)$ y otras formas que son absorbidas en menores cantidades. Una vez que el $\mathrm{P}$ es absorbido, este es distribuido a cada una de las células, concentrándose más en las partes reproductivas (Pineda-Mares et al., 2001).

La respuesta de los cultivos a la fertilización fosfatada depende del nivel de $\mathrm{P}$ disponible en el suelo, pero también es afectada por factores del suelo, del cultivo y de manejo del fertilizante. Entre los factores del suelo, se destacan la textura, la temperatura, el contenido de materia orgánica y el $\mathrm{pH}$; mientras que entre los del cultivo deben mencionarse los requerimientos y el nivel de rendimiento (García, 2001).

El P se caracteriza por su baja movilidad en el suelo y baja eficiencia de absorción por los vegetales y, como contrapartida, elevada residualidad hacia los cultivos subsiguientes. Esta característica hace que su manejo pueda realizarse bajo dos criterios de recomendación: el de suficiencia y el de reconstrucción y mantenimiento. El criterio de suficiencia pretende satisfacer los requerimientos del cultivo a implantar, esto es, aplicar la dosis óptima económica; mientras que el de reconstrucción y mantenimiento, incluye aportes para mantener o mejorar el nivel de $\mathrm{P}$ disponible en el suelo, pero además propone dosis que por lo común permiten obtener el rendimiento máximo (Ferraris, 2008).

Según estudios realizados por el Instituto de Investigación Agropecuaria de Panamá (IDIAP), se ha podido determinar que la mayoría de los suelos en Panamá tienen niveles bajos de fósforo disponible y se espera que den una respuesta positiva a la aplicación de este elemento (Name y Cordero, 1987; Gordon et al., 1995; Villarreal et al., 2013, Villarreal et al., 2015). Varios estudios para determinar la dosis óptima de este nutrimento en distintas localidades y tipos de suelo, encontraron que la dosis de $\mathrm{P}$ para el cultivo de maíz oscila entre 81 a 102 kg $\mathrm{P}_{2} \mathrm{O}_{5} /$ ha (Gordon et al., 1992). Todos estos trabajos fueron realizados con una densidad de planta de 5,33 plantas $/ \mathrm{m}^{2}$ en un arreglo de $0,75 \mathrm{~m}$ entre hileras y $0,50 \mathrm{~m}$ entre puntos de siembra, dejando dos plantas en cada punto. En la actualidad la dosis que se está recomendando para las siembras de este cultivo en el país es de $68 \mathrm{~kg} \mathrm{P}_{2} \mathrm{O}_{5} / \mathrm{ha}$ aplicados en banda al momento de la siembra, junto a los otros nutrimentos. Estudios recientes indican que el aumento de la densidad de plantas a 6,25 plantas $/ \mathrm{m}^{2}$ incrementó los rendimientos de grano en más de una tonelada por hectárea (Gordon et al., 2000).

Experimentos realizados por Bernal et al. (2014) en la región de sabanas ácidas de Colombia, encontraron respuestas lineales con la aplicación de hasta $90 \mathrm{~kg} \mathrm{P}_{2} \mathrm{O}_{5} /$ ha en suelos con contenido de $\mathrm{P}$ de $2 \mathrm{mg} / \mathrm{kg}$ y de aplicaciones de $60 \mathrm{~kg} \mathrm{P}_{2} \mathrm{O}_{5} /$ ha en suelos con bajo contenido de $\mathrm{P}$ de $14 \mathrm{mg} / \mathrm{kg}$.

En ensayos de fertilización fosfatada en el cultivo de maíz, realizados en la región serrana del sudeste de la Provincia de Buenos Aires durante los años 1994/95, 1995/96 y 1996/97, se observaron respuestas promedio de $790 \mathrm{~kg}$ de $\mathrm{P}_{2} \mathrm{O}_{5} /$ ha y significativas en siete de los ocho sitios con menos de $15 \mathrm{mg}$ de $\mathrm{P}$ disponible/ $\mathrm{kg}$ (P disponible promedio $=8 \mathrm{mg}$ de $\mathrm{P} / \mathrm{kg}$ ). El grupo de tres ensayos con más de $15 \mathrm{mg}$ de $\mathrm{P}$ disponible $/ \mathrm{kg}$ (P disponible promedio igual a $30 \mathrm{mg}$ de $\mathrm{P} / \mathrm{kg}$ ), mostró respuestas significativas en solo uno de ellos (Prystupa et al., 2006). Bordoli y Mallarino (1998) reportaron que durante veintiséis sitios-años en Iowa, Estados Unidos (750-1000 mm de precipitación anual) no hubo diferencias en rendimiento al utilizar tres diferentes formas de aplicación (al voleo superficial, bandeado profundo a $15-18 \mathrm{~cm}$ o bandeado $5 \mathrm{~cm}$ debajo y $5 \mathrm{~cm}$ al costado de la semilla) de P en maíz en sistemas de cero laboreos.

El objetivo del presente trabajo fue determinar, en el cultivo de maíz, el efecto del manejo de la fertilización fosforada a mediano y largo plazo con dos métodos de aplicación. 


\section{MATERIALES Y MÉTODOS}

Se estableció un ensayo en la Estación Experimental de El Ejido ubicada en la provincia de Los Santos a $7^{\circ} 58^{\prime}$ latitud norte y $80^{\circ} 22^{\prime}$ longitud oeste, en la segunda época de siembra (agosto-enero) durante diez años (2004 al 2013). La siembra fue realizada en un suelo de mediana fertilidad, en una parcela cuyo análisis inicial de suelo indicó una textura franco arcillosa, y pH muy ácido; sin embargo, no presentó problemas de alta saturación de aluminio, bajo en fósforo, contenido medio de potasio y materia orgánica y alto en calcio y magnesio, clasificado como un suelo Udic Haplustalf (Cuadro 1). Previo a la siembra de cada año, se tomó una muestra al azar en tres puntos de cada unidad experimental y se envió una muestra homogénea de la misma al laboratorio de suelos de IDIAP. Adicional a este muestreo previo a la siembra, durante los primeros siete años (2004 al 2010), se tomó una muestra a cada parcela a los sesenta días después de siembra (dds). Durante los primeros ocho años se utilizó el híbrido simple de maíz 30F-80 y en los últimos dos años el híbrido simple 30F-35.

El diseño experimental utilizado fue de bloques completos al azar con tres repeticiones. Se evaluaron dos factores en un factorial 5 x 4 con un arreglo de fajas. El modelo estadístico para el análisis a través de los años fue el siguiente:

$\gamma_{i j k l}=\mu+\alpha_{i}+\rho_{j}+\gamma_{k}+(\alpha \gamma)_{i k}+\sigma_{i j k}+\beta_{l}+(\alpha \beta)_{i l}+\tau_{i j l}+\gamma \beta_{k l}+(\alpha \gamma \beta)_{i k l}+\varepsilon_{i j k}$

En donde:

$\mu=$ Media general del experimento

$\alpha_{\mathrm{i}}=$ Efecto de años

$\mathrm{Q}_{\mathrm{j}}=$ Efecto de repeticiones

$\gamma_{\mathrm{k}}=$ Efecto de la aplicación al voleo del P $\alpha \gamma_{i \mathrm{ik}}=$ Interacción año $\mathrm{x}$ voleo

$\delta_{i \mathrm{ik}}=$ Error I (año x voleo x repetición)

$\beta_{1}=$ Efecto de la aplicación en banda de $\mathrm{P}$

$\alpha \beta_{\mathrm{il}}=$ Interacción año $\mathrm{x}$ banda

$\tau_{\mathrm{ijl}}=$ Error II (año $\mathrm{x}$ banda $\mathrm{x}$ repetición)

$\gamma \beta_{\mathrm{k} 1}=$ Interacción voleo $\mathrm{x}$ banda

$\alpha \gamma \beta_{\mathrm{ikl}}=$ Interacción año $\mathrm{x}$ voleo $\mathrm{x}$ banda

$\varepsilon_{\mathrm{ijkl}}=$ Error residual

A las distintas variables medidas se les realizó un análisis utilizando el procedimiento "PROC MIXED" en donde la repetición se consideró variable al azar, las aplicaciones al voleo y banda como factores fijos y los años fueron considerados como medidas repetidas de acuerdo con Littell et al. (2006). Para la estructura de las covarianzas se utilizó el modelo no estructurado (Unstructured). A todas las variables se le calculó las medias ajustadas (lsmeans) y las mismas fueron comparadas utilizando las diferencias mínimas significativas (DMS) al 5\% de probabilidad.

La parcela principal (fajas) la constituyó la aplicación de fósforo al voleo, en dosis de 0, 25, 50, 75 y $100 \mathrm{~kg}$ P/ha. Las sub-parcelas consistieron en las aplicaciones de fósforo en banda, en dosis de 0, 10, 20 y $30 \mathrm{~kg} \mathrm{P} / \mathrm{ha}$. El fósforo al voleo fue aplicado antes de la siembra e incorporado a $15 \mathrm{~cm}$ de profundidad mediante una rastra el primer año (2004). El fósforo en banda fue aplicado al momento de la siembra junto con los otros nutrimentos cada año. En total se evaluaron veinte tratamientos (Cuadro 2). Con esta distribución se logró aplicar desde 0 a $400 \mathrm{~kg} \mathrm{P} / \mathrm{ha}$ en los distintos tratamientos evaluados a través de los diez años que duró el estudio.

La fuente de fósforo utilizada fue súper fosfato triple (0-46-0). En este experimento los tratamientos de 0 voleo más 0 banda (T1) y 0 voleo más 30 banda (T4) fueron considerados como testigo absoluto y

Cuadro 1. Análisis de suelo de la parcela experimental previo a la instalación del experimento para determinar el efecto del manejo de la fertilización fosforada en maíz. El Ejido, Panamá. 2004.

Table 1. Soil analysis from experimental plot before trial installation to determine the effect of phosphorus management in corn. El Ejido, Panama. 2004.

\begin{tabular}{|c|c|c|c|c|c|c|c|c|c|c|c|c|c|c|}
\hline & Arena & Limo & Arcilla & pH & $\mathbf{P}$ & $\mathbf{K}$ & $\mathbf{C a}$ & Mg & Al & M.O. & Mn & $\mathrm{Fe}$ & $\mathbf{Z n}$ & $\mathrm{Cu}$ \\
\hline & & $\%$ & & $1: 2.5$ & $\mathrm{mg} / \mathrm{kg}$ & ---.- & $-\mathrm{cmo}$ & +) $/ \mathbf{k g ~ - -}$ & -.---- & $\%$ & \multicolumn{4}{|c|}{--------mg/kg-------- } \\
\hline Media & 37 & 21 & 42 & 4,4 & 1,33 & 0,20 & 6,67 & 5,05 & 0,18 & 4,43 & 40,3 & 5,9 & 3,3 & 3,8 \\
\hline Int. confianza $95 \%$ & 1,3 & 0,7 & 1,4 & 0,1 & 0,10 & 0,03 & 0,23 & 0,35 & 0,03 & 0,25 & 2,29 & 0,5 & 2,9 & 0,2 \\
\hline
\end{tabular}


Cuadro 2. Estructura de los tratamientos del ensayo para el manejo de la fertilización fosforada en el cultivo de maíz. El Ejido, Panamá, 2004-2013.

Table 2. Trial treatment structure of phosphorus fertilization management in corn crop. El Ejido, Panama. 20042013.

\begin{tabular}{|c|c|c|c|c|c|c|c|}
\hline Tratamiento & $\begin{array}{l}2004 \\
\text { voleo } \\
\mathrm{kg} / \mathrm{ha}\end{array}$ & $\begin{array}{l}\text { 2004-2013 } \\
\text { Banda/año }\end{array}$ & $\begin{array}{c}\text { Total P } \\
\text { kg/ha }\end{array}$ & Tratamiento & $\begin{array}{l}2004 \\
\text { voleo } \\
\mathrm{kg} / \mathrm{ha}\end{array}$ & $\begin{array}{c}\text { 2004-2013 } \\
\text { banda/año } \\
\text { kg/ha }\end{array}$ & $\begin{array}{c}\text { Total P } \\
\text { aplicado } \\
\text { kg/ha }\end{array}$ \\
\hline 1 & 0 & 0 & 0 & 11 & 50 & 20 & 250 \\
\hline 2 & 0 & 10 & 100 & 12 & 50 & 30 & 350 \\
\hline 3 & 0 & 20 & 200 & 13 & 75 & 0 & 75 \\
\hline 4 & 0 & 30 & 300 & 14 & 75 & 10 & 175 \\
\hline 5 & 25 & 0 & 25 & 15 & 75 & 20 & 275 \\
\hline 6 & 25 & 10 & 125 & 16 & 75 & 30 & 375 \\
\hline 7 & 25 & 20 & 225 & 17 & 100 & 0 & 100 \\
\hline 8 & 25 & 30 & 325 & 18 & 100 & 10 & 200 \\
\hline 9 & 50 & 0 & 50 & 19 & 100 & 20 & 300 \\
\hline 10 & 50 & 10 & 150 & 20 & 100 & 30 & 400 \\
\hline
\end{tabular}

testigo del productor, respectivamente. Cada parcela experimental constó de seis surcos de 5,2 m de largo a una densidad teórica inicial de 6,25 plantas $/ \mathrm{m}^{2}$. La parcela efectiva fueron los dos surcos centrales de cada unidad experimental. Todos los tratamientos recibieron por año una fertilización base de $200 \mathrm{~kg}$ de $\mathrm{N} / \mathrm{ha}$, se utilizó como fuente la urea $(46 \% \mathrm{~N})$ con el siguiente fraccionamiento: $10 \%$ a la siembra, $40 \%$ a los veinte días después de la siembra (dds) y $50 \%$ a los 37 dds (Gordon et al., 2004). También se le aplicó $20 \mathrm{~kg}$ de $\mathrm{K}_{2} \mathrm{O} /$ ha más $20 \mathrm{~kg} \mathrm{~S} /$ ha al momento de la siembra.

Se le realizó un análisis de regresión Linear Plateau para determinar el nivel crítico de fósforo en el suelo, además de regresiones cuadráticas para determinar la dosis de $\mathrm{P}$ recomendada. Se tomaron datos de peso de campo de la mazorca, número de plantas y mazorcas cosechadas, porcentaje de humedad del grano, rendimiento de rastrojo, número de plantas acamadas y altura de planta. Se realizó un análisis de suelo antes de la siembra. Para la determinación del fósforo en las muestras de suelo se utilizó el método de extracción de Mehlich 1, para el resto de los análisis se utilizó la metodología descrita por Díaz-Romeu y Hunter (1982). Se calcularon los componentes del rendimiento estándar (Bolaños y Barreto, 1991), así como el rendimiento relativo por sistema de voleo, en donde el $100 \%$ es el valor máximo entre todos los rendimientos por cada año. Se midió la precipitación pluvial en el período de desarrollo del cultivo cada año. Los datos de lluvia se agruparon en los cuatro períodos críticos descritos por McWilliam et al. (1999); estos autores agrupan a las fases fenológicas del cultivo en fase de establecimiento ( 0 a $30 \mathrm{dds}$ ), pre floración (31 a 50 dds), floración y llenado de grano (51 a 80 dds) y fase final de llenado de grano (81 a 100 dds). Se realizó un análisis económico del combinado de los diez años utilizando la metodología de presupuesto parcial de Jauregui y Saín (1990).

\section{RESULTADOS Y DISCUSIÓN}

\section{Análisis estadístico}

El análisis de varianza combinado de los diez años indicó diferencias altamente significativas entre años para todas las variables medidas. El factor voleo presentó diferencias estadísticas (significativas y altamente significativas) para todas las variables con excepción del número de plantas cosechadas. La aplicación en bandas también mostró diferencias altamente significativas para todas las variables con excepción del número de plantas cosechadas, peso de mazorcas, porcentaje de plantas acamadas y contenido de $\mathrm{P}$ en el suelo (Cuadro 3). En relación con la interacción voleo por banda solo se encontró 
Cuadro 3. Valor del estadístico $\mathrm{F}$ del análisis de varianza para las variables de rendimiento de grano y otras características agronómicas para determinar el efecto de la fertilización fosforada en maíz. El Ejido, Panamá. 2004-2013.

Table 3. F value from variance analysis table for grain yield and other agronomic characteristics variables to determine the effect of phosphorus management in corn. El Ejido, Panama. 2004-2013.

\begin{tabular}{lcccccccccc}
\hline F. de V & g.l. & $\begin{array}{c}\text { Rend. } \\
\text { Grano }\end{array}$ & $\begin{array}{c}\text { Rend. } \\
\text { Rastrojo }\end{array}$ & Biomasa & $\begin{array}{c}\text { Rend. } \\
\text { relativo }\end{array}$ & $\begin{array}{c}\text { Peso } \\
\text { mazorca }\end{array}$ & $\begin{array}{c}\text { Planta/ } \\
\mathbf{m}^{2}\end{array}$ & $\begin{array}{c}\text { Mazorca/ } \\
\mathbf{m}^{2}\end{array}$ & $\begin{array}{c}\text { Mazorca/ } \\
\text { planta }\end{array}$ & $\begin{array}{c}\text { Contenido } \\
\text { de P }\end{array}$ \\
\hline Año & 9 & $280,1^{* *}$ & $151,3^{* *}$ & $211,1^{* *}$ & $46,2^{* *}$ & $129,6^{* *}$ & $202,7^{* *}$ & $81,5^{* * *}$ & $23,2^{* *}$ & $143,28^{* *}$ \\
Voleo & 4 & $11,4^{* *}$ & $5,9^{*}$ & $8,7^{* *}$ & $15,4^{* *}$ & $16,9^{* *}$ & $0,4^{\text {n.s. }}$ & $3,8^{* *}$ & $5,6^{* *}$ & $2,89 *$ \\
Banda & 3 & $33,2^{* *}$ & $5,9^{* *}$ & $14,0^{* *}$ & $25,2^{* *}$ & $23,1^{\text {n.s. }}$ & $1,4^{\text {n.s. }}$ & $10,1^{* *}$ & $8,1^{* *}$ & $1,18^{\text {n.s. }}$ \\
Voleo x banda & 12 & $2,1^{* *}$ & $0,5^{\text {n.s. }}$ & $0,9^{\text {n.s. }}$ & $1,8^{\text {n.s. }}$ & $2,0^{*}$ & $0,3^{\text {n.s. }}$ & $1,1^{\text {n.s. }}$ & $1,6^{\text {n.s. }}$ & $0,92^{\text {n.s. }}$ \\
Año x voleo & 36 & $5,7^{* *}$ & $3,8^{* *}$ & $5,0^{* *}$ & $5,5^{* *}$ & $6,9^{* *}$ & $2,3^{* *}$ & $1,5^{\text {n.s. }}$ & $1,8^{*}$ & $9,12^{*}$ \\
Año x banda & 27 & $9,2^{* *}$ & $5,8^{* *}$ & $8,2^{* *}$ & $7,7^{* *}$ & $11,0^{* *}$ & $1,9^{*}$ & $2,8^{* *}$ & $2,7^{* *}$ & $1,90 *$ \\
Año x voleo x & 108 & $1,8^{* *}$ & $1,7^{* *}$ & $1,5^{* *}$ & $1,9^{* *}$ & $1,5^{\text {n.s. }}$ & $1,5^{\text {n.s. }}$ & $1,4^{\text {n.s. }}$ & $1,6^{*}$ & 1,20 n.s. \\
banda & & & & & & & & & & \\
BCI & & 933,4 & 1358,2 & 1595,9 & $-614,1$ & 3334,9 & 563,1 & 810,2 & $-822,8$ & 2118,5 \\
\hline
\end{tabular}

**** Diferencias estadísticas al 1 y 5\%, respectivamente, ${ }^{\text {n.s. }}$ no hay diferencias significativas / Statistical differences at 1 and $5 \%$, respectively, ${ }^{\text {n.s. }}$ no significant differences.

$\mathrm{BCI}=$ Criterio de información Bayesiano $/ \mathrm{BCI}=$ Bayesian criterion information .

diferencias altamente significativas para el rendimiento de grano. Esta variable también presentó interacción significativa para el análisis de año $\mathrm{x}$ voleo, año $\mathrm{x}$ banda y año $\mathrm{x}$ voleo $\mathrm{x}$ banda. La variable número de plantas al momento de la cosecha solo presentó diferencias estadísticas para la fuente de variación que incluyeron el factor año.

\section{Análisis del contenido de fósforo en el suelo}

Luego del primer año de evaluación el contenido de $\mathrm{P}$ disminuyó en todas las parcelas, principalmente en los tratamientos al voleo de 75 y $100 \mathrm{~kg} \mathrm{P} / \mathrm{ha}$ (Figura 1). De acuerdo con el resultado de análisis de $\mathrm{P}$ según Mehlich 1, a partir del quinto año de evaluación (2008) no se encontró diferencias en el contenido de $\mathrm{P}$ en el suelo (diferencia mínima significativa de $2,01 \mathrm{mg}$ ) entre ninguna de las franjas de $\mathrm{P}$ al voleo (contenido menor de $5 \mathrm{mg} \mathrm{P} / \mathrm{dm}^{3}$ ). Sin embargo, la respuesta en rendimiento de grano mostró diferencias significativas entre los niveles de $\mathrm{P}$ aplicados al voleo. Este resultado sugiere que el método Mehlich 1, no está extrayendo el $\mathrm{P}$ que está en el suelo disponible para la planta, a pesar de que el $\mathrm{pH}$ del suelo es menor a 5,0, este resultado contrasta con lo expresado por algunos investigadores que afirman que el análisis

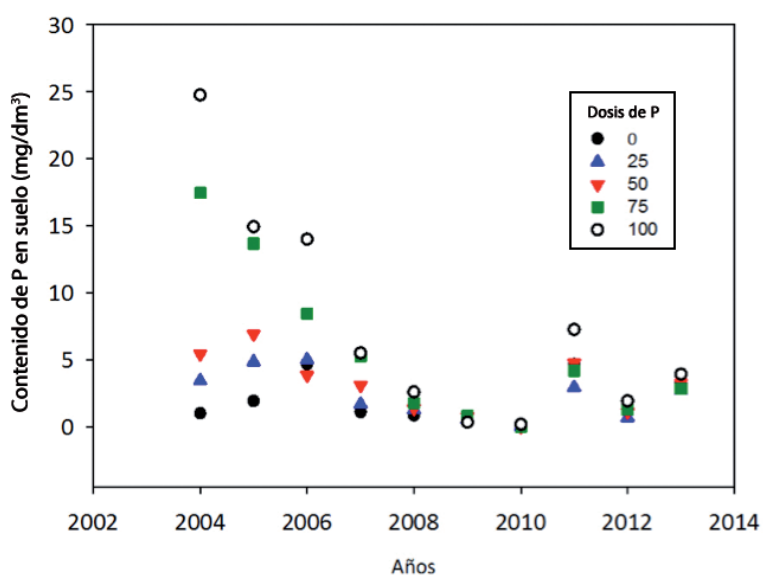

Figura 1. Contenido de fósforo en el suelo (Mehlich 1) por año en las bandas según dosis de aplicación al voleo. El Ejido, Panamá. 2004-2013.

Figure 1. Phosphorus soil content (Mehlich 1) per year in bands according broadcast application rate. El Ejido, Panama. 2004-2013.

de suelo con el método Mehlich 1 se adapta mejor a los suelos ácidos $(\mathrm{pH}<5,5)$ con escasa capacidad de intercambio catiónico $(<10 \mathrm{cmol} / \mathrm{kg})$ y con contenido de materia orgánica menor al 5\% (Pérez, 1995; Colpo- 
Gatiboni et al., 2010). Esto sugiere que se deben realizar nuevos trabajos con diferentes soluciones extractoras (Mehlich-3, Bray 1 y 2, Olsen, etc.) para encontrar una metodología que determine la presencia de este elemento en los análisis rutinarios de suelo.

El ajuste del modelo Linear Plateau de los datos de rendimiento de grano y los niveles de $\mathrm{P}$ medidos en los tratamientos al voleo, los análisis de suelo en las distintas franjas de $\mathrm{P}$ aplicadas al voleo el primer año, de los tres primeros años del experimento, fue significativo con un $\mathrm{R}^{2}$ de 0,85 . El valor crítico o punto de quiebre de la curva para la recomendación de la aplicación de P fue de $13,3 \mathrm{mg} / \mathrm{dm}^{3}$, es decir que en suelos con contenido mayores a este es poco probable que haya respuesta a la aplicación de este elemento (Figura 2). Este valor crítico es menor que el reportado por Name y Cordero (1987) en donde los mismos indicaban un valor de 18 $\mathrm{mg} / \mathrm{dm}^{3}$, y similar al reportado por Ramírez et al. (1988) quienes sugirieron que valores entre 11 y $14 \mathrm{mg} / \mathrm{dm}^{3}$ era el nivel crítico para que la respuesta a la aplicación de este nutrimento fuera significativa.

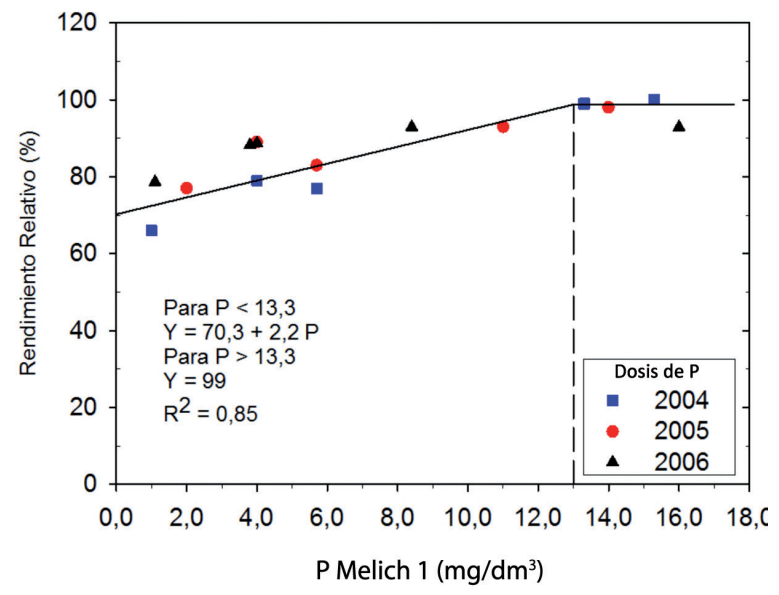

Figura 2. Curva de respuesta Linear-Plateau entre el contenido de fósforo en el suelo y el rendimiento relativo de maíz. El Ejido, Panamá. 2004-2006.

Figure 2. Linear-Plateau curve response between phosphorus soil content and relative maize yield. El Ejido, Panama. 2004-2006.

\section{Análisis de los componentes del rendimiento}

El análisis estadístico de las variables que componen el rendimiento tales como número de plantas y mazorcas, peso de mazorcas y rendimiento de rastrojo indicaron que la mayor variabilidad estuvo en la fuente de variación entre años, presentado todas diferencias altamente significativas (Cuadro 4). El año 2004 fue el más afectado tanto en el número de plantas y mazorcas por metro cuadrado así como de mazorcas cosechadas por planta. En relación con el número de mazorcas cosechadas por planta, a partir del año 2011 se presentó una disminución de los valores obtenidos (menos de 0,94 mazorcas/planta) producto de la merma en el contenido de $\mathrm{P}$ en el suelo. En relación con el contenido de biomasa total, el mayor rendimiento se obtuvo en el 2010 seguido por el obtenido en 2005 con rendimientos superiores a las 14 t/ha. El análisis de la interacción voleo por banda no fue significativa para ninguno de los componentes del rendimiento excepto el de peso de mazorca, mismo que será discutido junto a la variable rendimiento de grano. El resto de las interacciones de las variables analizadas presentaron diferencias estadísticas muy relacionadas por el efecto de los años.

\section{Efecto de años}

Los dos híbridos utilizados en este ensayo son considerados intermedios con una floración femenina entre 52 a 54 dds y la formación de la capa negra (madurez fisiológica) entre los 100 a 110 dds. El rendimiento de grano de todos los tratamientos por ciclo de evaluación durante todo el período del estudio varió entre 4,12 a 8,75 t/ha (Cuadro 4). El análisis estadístico indicó diferencias altamente significativas entre años, siendo este el factor que mayor contribución dio a la variabilidad encontrada en los análisis de todas las variables. El valor de la diferencia mínima significativa (DMS) al 5\% de probabilidad fue de $0,35 \mathrm{t} / \mathrm{ha}$.

Con excepción del primer año (2004) la siembra del ensayo se realizó entre finales de agosto a principios de septiembre. La variable que explicó esta variación a través de los años fue la precipitación acumulada en las distintas fases de desarrollo del cultivo. En el año 2004 el rendimiento fue severamente afectado por el estrés hídrico en las últimas tres etapas del cultivo (31 a 50,51 a 80 y más de 80 dds) con $99,0,57,2$ y 0,0 $\mathrm{mm}$ de lluvia para cada período, respectivamente. En el año 2008 el estrés ocurrió en el segundo período (51 a 80 dds) con $61,0 \mathrm{~mm}$, mientras que en el 2011 fue afectado entre los 31 y 50 dds. En los años 2006 
Cuadro 4. Número de plantas y mazorcas cosechadas, peso de mazorcas y rendimiento de grano (14\% humedad), forraje (materia seca) y biomasa total por año en parcelas con adición de fósforo al voleo y en bandas. El Ejido, Panamá. 2004-2013.

Table 4. Number of plant and ear harvested, ear weight and grain yield (14\% moisture), foliage yield and total biomass by year, in plots with phosphorus broadcast and in bands. El Ejido, Panama. 2004-2013.

\begin{tabular}{lccccccc}
\hline Año & $\begin{array}{c}\text { Plantas/ } \\
\mathbf{m}^{2}\end{array}$ & $\begin{array}{c}\text { Mazorcas/ } \\
\mathbf{m}^{2}\end{array}$ & $\begin{array}{c}\text { Mazorca/ } \\
\text { planta }\end{array}$ & $\begin{array}{c}\text { Peso mazorca } \\
(\mathbf{g})\end{array}$ & $\begin{array}{c}\text { Rend. forraje } \\
(\mathbf{t} / \mathbf{h a})\end{array}$ & $\begin{array}{c}\text { Rend grano } \\
(\mathbf{t} / \mathbf{h a})\end{array}$ & $\begin{array}{c}\text { Biomasa } \\
(\mathbf{t} / \mathbf{h a})\end{array}$ \\
\hline 2004 & 4,68 & 3,83 & 0,82 & 103 & 5,00 & 4,00 & 8,44 \\
2005 & 6,09 & 5,78 & 0,95 & 136 & 7,29 & 7,87 & 14,06 \\
2006 & 5,77 & 5,73 & 0,99 & 121 & 6,06 & 6,92 & 12,01 \\
2007 & 6,15 & 6,04 & 0,98 & 130 & 6,71 & 7,83 & 13,44 \\
2008 & 5,51 & 5,26 & 0,96 & 130 & 7,33 & 6,86 & 13,23 \\
2009 & 5,63 & 5,45 & 0,97 & 126 & 6,51 & 6,84 & 12,39 \\
2010 & 6,05 & 5,89 & 0,97 & 149 & 9,36 & 8,75 & 16,88 \\
2011 & 5,82 & 5,36 & 0,92 & 128 & 6,08 & 6,89 & 12,00 \\
2012 & 6,41 & 5,73 & 0,89 & 99 & 6,46 & 5,78 & 11,43 \\
2013 & 5,88 & 5,48 & 0,93 & 117 & 5,67 & 6,49 & 11,25 \\
Prom & 5,80 & 5,46 & 0,94 & 124 & 6,65 & 6,82 & 12,51 \\
DMS & 0,12 & 0,21 & 0,03 & 5 & 0,42 & 0,35 & 0,65 \\
\hline
\end{tabular}

y 2009, el estrés se presentó tanto en la fase de 31 a 50 dds $(57,0 \mathrm{~mm})$ como en la fase de más de 80 dds $(45,0 \mathrm{~mm})$.

En el 2012 y 2013 el estrés se presentó después de los 80 dds. En el 2005, 2007 y 2010 el desarrollo del cultivo fue normal y no fue sometido a ningún tipo de estrés hídrico. La precipitación acumulada entre los 80 a 100 dds presentó el mayor valor del coeficiente de determinación $\left(\mathrm{R}^{2}\right.$ de 0,89$)$, seguida por la precipitación acumulada entre los 51 a 80 dds $\left(R^{2}\right.$ de 0,46$)$. En la Figura 3a y 3 b se aprecia la relación de las dos variables estudiadas. El efecto del estrés hídrico en estas etapas coincide con lo reportado por McWilliams et al. (1999), en donde las fases de floración y llenado del grano (51 a 80 dds) son más susceptibles y se ven reflejadas en la merma de la producción de grano. El número de plantas al momento de la cosecha varió entre 4,69 a 6,15 plantas $/ \mathrm{m}^{2}$, siendo el año 2004 el que mostró una menor población de plantas. Investigaciones conducidas por Senigagliesi et al. (1984) durante la década del 80, encontraron relaciones significativas entre el nivel de $\mathrm{P}$ disponible a la siembra y la respuesta a la fertilización fosfatada en maíz. Además, determinaron umbrales críticos de $\mathrm{P}$ e indicaron que las respuestas se reducían si ocurrían déficits hídricos en el período crítico prefloración a floración.

\section{Respuesta a la aplicación de fósforo}

La Figura 4 presenta la respuesta cuadrática del rendimiento acumulado vs la cantidad de $\mathrm{P}$ aplicado a cada una de las parcelas (suma de la aplicación al voleo más la aplicación en banda anual). De acuerdo con la ecuación de regresión, el máximo físico se logró con la aplicación de $304 \mathrm{~kg}$ P/ha (media de 30,4 kg/año) coincidiendo este valor con el reportado por Bernal et al. (2014) en Colombia; este valor coincide con el recomendado por el Instituto a los productores de la región de Azuero (Gordón et al., 1995).

Las diferencias mínimas significativas entre tratamientos variaron a través de los años en función del potencial de rendimiento obtenido en cada año. El máximo rendimiento se obtuvo con la aplicación inicial de $75 \mathrm{~kg}$ al voleo más la aplicación de $30 \mathrm{~kg} / \mathrm{año}$ (T16) con una media de 7,77 t/ha. Los tratamientos a base de 100 kg P al voleo (T18, T19 y T20), así como la aplicación de $75 \mathrm{~kg}$ al voleo y $20 \mathrm{~kg}$ en banda de $\mathrm{P} / \mathrm{ha}$ por año (T15) tuvieron rendimientos superiores a 7,57 t/ha, $y$ 

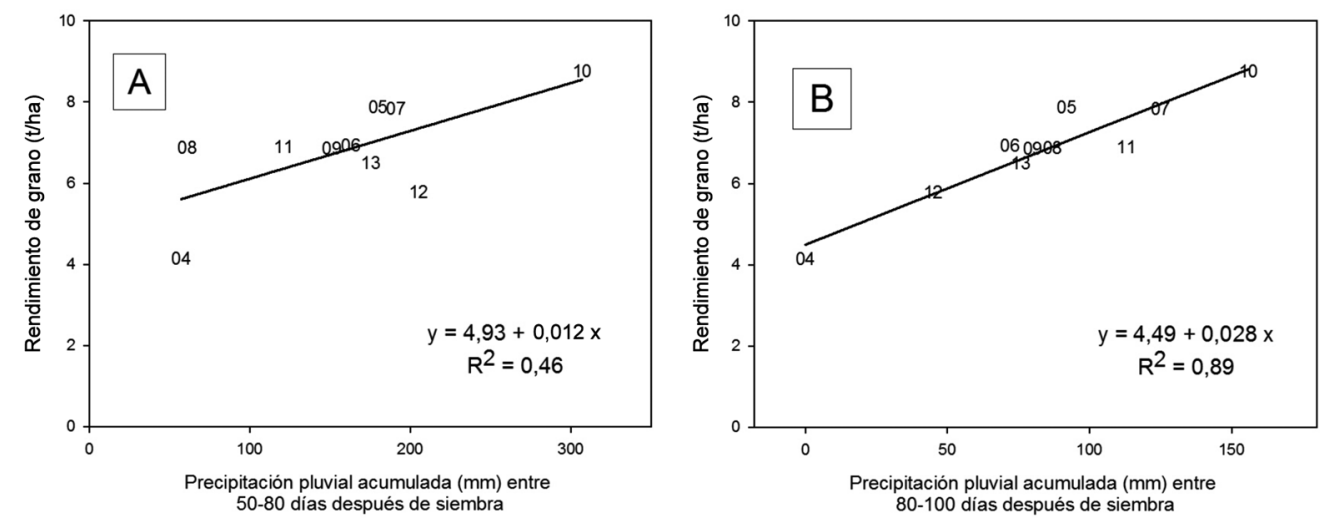

Figura 3. Relación entre el rendimiento de grano (t/ha) de maíz y la precipitación acumulada (mm) en los periodos entre 50-80 (A) y 80-100 días después de siembra (B). El Ejido, Panamá. 2004-2013.

Figure 3. Relation between grain yield (t/ha) and rainfall accumulated $(\mathrm{mm})$ in the stages $50-80(\mathrm{~A})$ and 80 100 days after planting (B). El Ejido, Panama. 2004-2013.

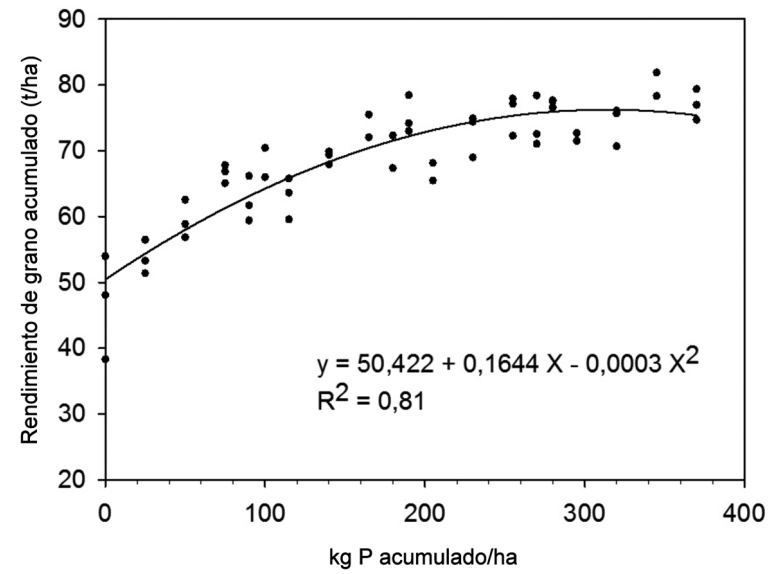

Figura 4. Curva de regresión cuadrática del rendimiento de grano de maíz en función del fósforo aplicado en los diez años de evaluación. El Ejido, Panamá. 2004-2013.

Figure 4. Regression quadratic curve from gain yield by phosphorus dosages applied during ten years of trial. El Ejido, Panama. 2004-2013.

no difirieron estadísticamente del tratamiento de mayor rendimiento, los cuales componen el grupo superior.

La Figura 5A y 5B muestran los promedios de los niveles de $\mathrm{P}$ al voleo y bandeado a través de los años. Se observa que el aumento en la dosis de fósforo al voleo aumentó el rendimiento de grano de 6,28 a 7,52 t/ha y el aumento de la dosis aplicada en banda incrementó el rendimiento de 5,95 a 7,44 t/ha. En ambos tipos de aplicación se observó una relación lineal del efecto del aumento de dosis. Los tratamientos de 0 y $25 \mathrm{~kg}$ $\mathrm{P} /$ ha al voleo no difirieron significativamente entre sí, pero las dosis de 50,75 y $100 \mathrm{~kg} \mathrm{P} /$ ha si presentaron diferencias estadísticas entre cada una de ellas. En relación con la aplicación en banda, el aumento de cada dosis significó ganancias estadísticas entre cada una. Welch et al. (1966) en estudios realizados en tres localidades en los Estados Unidos, informaron una respuesta creciente del maíz a dosis de fósforo en ambos sistemas de aplicación de este nutrimento. Esto sugiere que la dosis de mejor respuesta fue la de la aplicación de $30 \mathrm{~kg}$ P/ha en banda.

\section{Interacción de la aplicación al voleo vs banda}

El análisis estadístico de los datos indicó que la interacción entre dosis al voleo y banda fue altamente significativa; en las franjas con dosis de 0 y $25 \mathrm{~kg} / \mathrm{ha}$ hay diferencias entre las distintas dosis aplicadas en banda (Figura 6). Al observar la respuesta cuando se aplicó $50 \mathrm{~kg} / \mathrm{ha}$, la diferencia entre 20 y $30 \mathrm{~kg} / \mathrm{ha}$ no fue significativa, mientras que la aplicación de $10 \mathrm{~kg} / \mathrm{ha}$ 

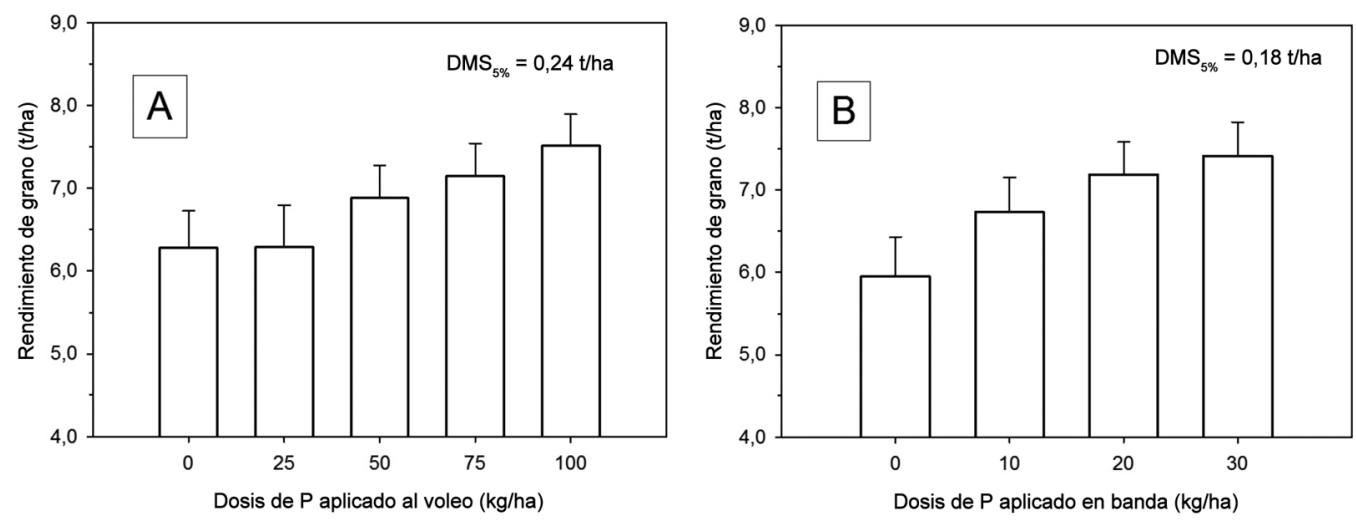

Figura 5. Rendimiento de grano de maíz (t/ha) según dosis de fósforo aplicada al voleo (A) y en banda (B) a través de diez años de evaluación. El Ejido, Panamá. 2004-2013.

Barras verticales representan DMS $(\mathrm{P}<0,05)$.

Figure 5. Corn grain yield (t/ha) by phosphorus dosages broadcast applied (A) and band (B) through 10 year of evaluation. El Ejido, Panama. 2004-2013. Vertical bars represent LSD $(\mathrm{P}<0.05)$.

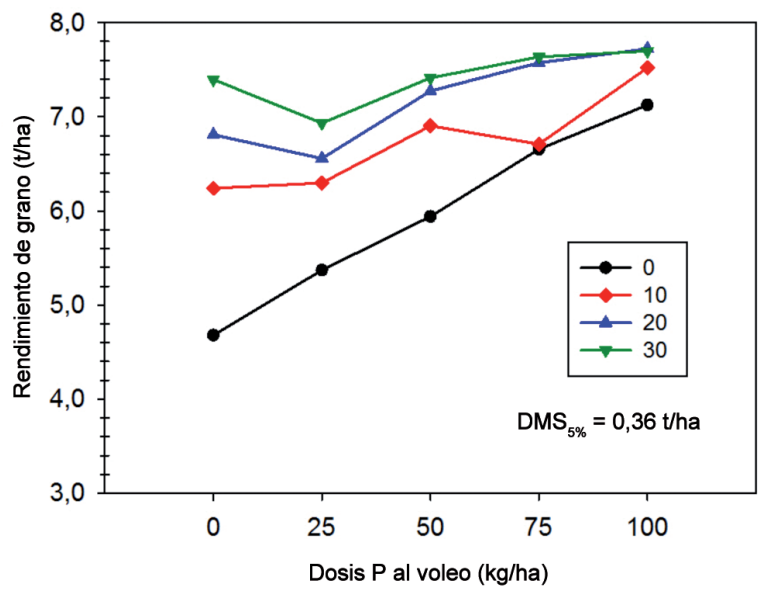

Figura 6. Respuesta del rendimiento de grano de maíz por la interacción entre dosis al voleo y dosis por banda de fósforo a través de diez años de evaluación. El Ejido, Panamá. 2004-2013.

Figure 6. Grain yield response by the interaction between broadcast and band dosages through 10 years of evaluation. El Ejido, Panama. 2004-2013.

superó a las parcelas sin aplicación de fósforo. Por otro lado, en la franja que se aplicó $100 \mathrm{~kg} / \mathrm{ha}$ al voleo no hubo diferencias estadísticas entre los tres tratamientos aplicados con fósforo y la diferencia de estas con respecto a la no aplicación de fósforo fue mínima $(0,40$ t/ha). Estos resultados contrastan con lo reportado por Bordoli y Mallarino (1998), en donde no encontraron diferencias entre aplicar el fósforo en banda debajo de la semilla y las aplicaciones al voleo de este nutrimento.

\section{Interacción año $\mathrm{x}$ dosis al voleo $\mathrm{y}$ año $\mathrm{x}$ dosis en banda}

La interacción de aplicación al voleo por año (Figura 7A) fue estadísticamente significativa y el resultado obtenido indicó que en los primeros siete años del estudio (2004 a 2010) las diferencias entre las distintas dosis aplicadas fue mínima. A partir del año 2011 las diferencias entre dosis aumentaron para el año 2013, la dosis de $100 \mathrm{~kg} /$ ha superó al resto en al menos una tonelada. En relación con la interacción años x dosis en banda (Figura 7B) la respuesta encontrada fue similar a la interacción con dosis al voleo. En la misma se apreció una diferencia marcada entre dosis a partir del año 2011, principalmente en las parcelas sin aplicación de fósforo el rendimiento fue la mitad de lo obtenido con la aplicación de 20 y $30 \mathrm{~kg} / \mathrm{ha}$. Estos resultados contrastan con los obtenidos por Howard et al. (2002) en donde se obtuvieron resultados inconsistentes a través de los años en un estudio que duró diez años bajo siembra directa sin preparar el suelo. 

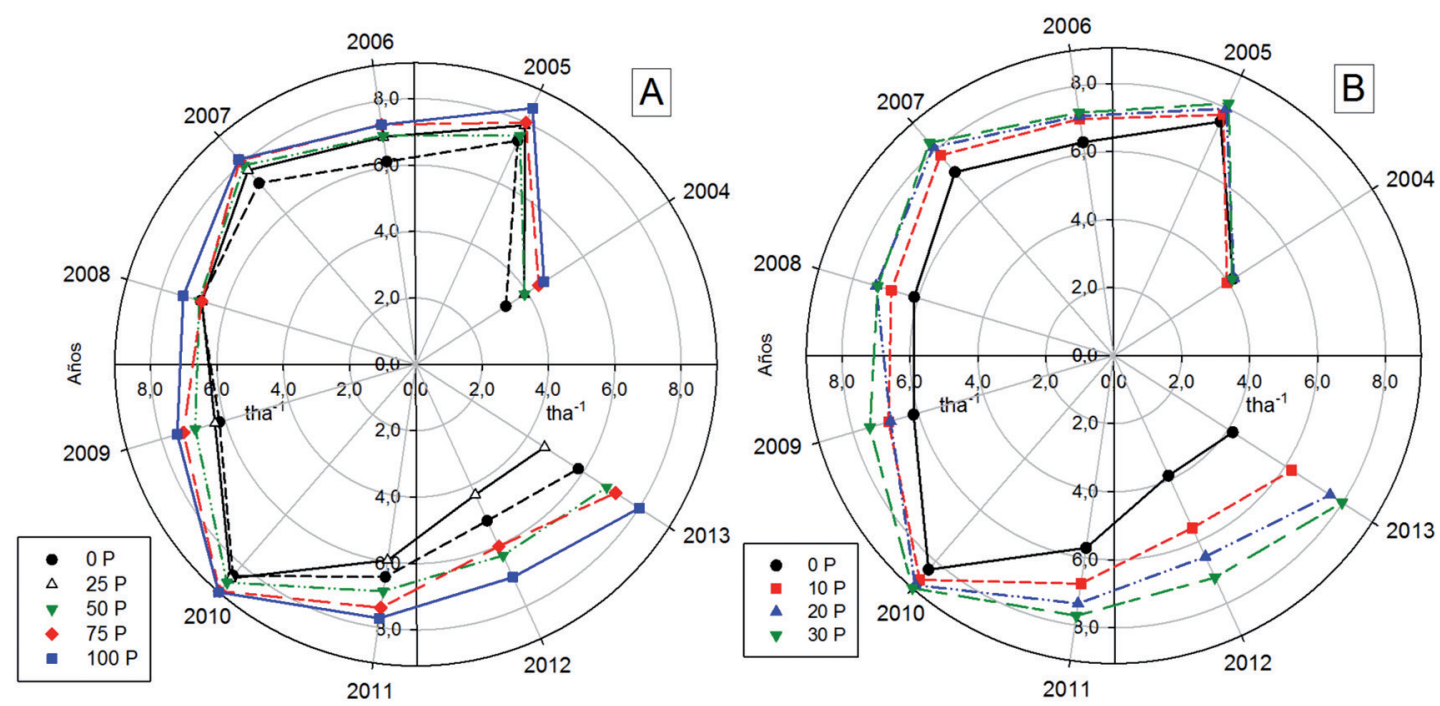

Figura 7. Interacción de la aplicación de dosis de fósforo al voleo vs año (A) y la aplicación de dosis de fósforo en banda vs año (B) en el rendimiento de grano de maíz. El Ejido, Panamá. 2004-2013.

Figure 7. Interaction phosphorus broadcast dosages vs year (A) and phosphorus band dosages vs year (B) of corn grain yield. El Ejido, Panama. 2004-2013.

\section{Efecto residual de las aplicaciones al voleo}

En los primeros siete años del estudio se encontró que el rendimiento relativo de los tratamientos al voleo permaneció estable; sin embargo, hubo diferencias significativas entre los mismos (Figura 8A). En este período los tratamientos de 100 y $75 \mathrm{~kg} / \mathrm{ha} \sin$ aplicación en banda fueron similares al rendimiento relativo del testigo del proyecto de IDIAP que fue superior al $90 \%$. El tratamiento de $50 \mathrm{~kg} / \mathrm{ha}$ se mantuvo alrededor del $85 \%$ de rendimiento relativo, mientras que el tratamiento de $25 \mathrm{~kg} / \mathrm{ha}$ disminuyó un $10 \%$
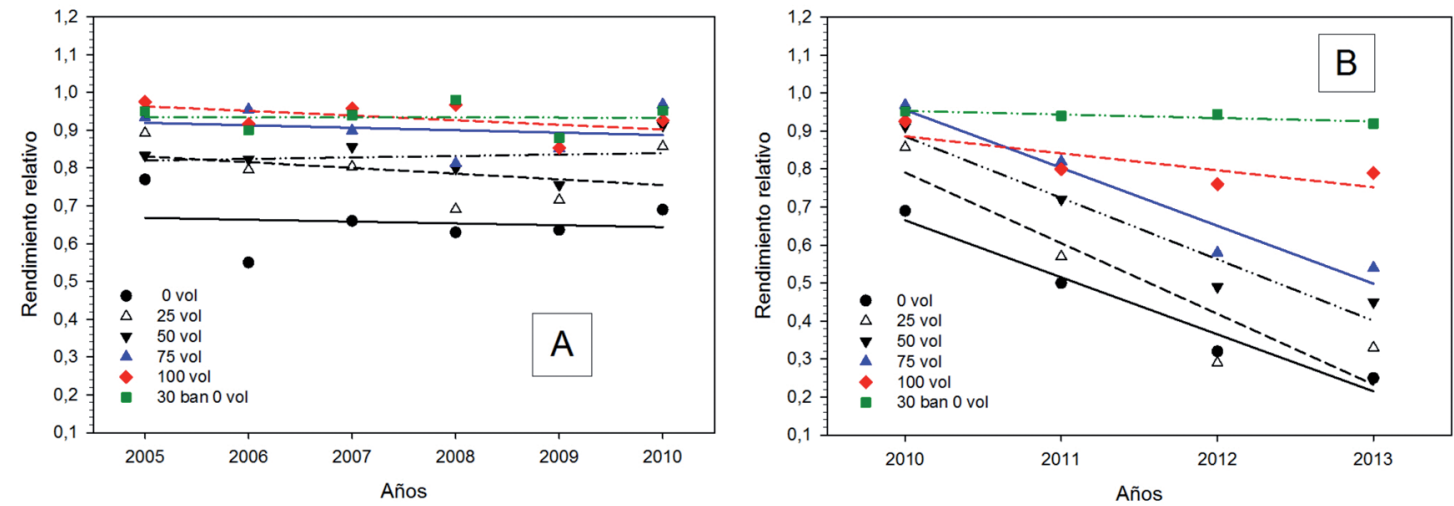

Figura 8. Rendimiento relativo a través de los años de las distintas dosis de fósforo aplicadas al voleo y el testigo del productor. El Ejido, Panamá, 2004-2013.

Figure 8. Relative yield through years of different broadcast doses of phosphorus applied and farmer tester. El Ejido, Panama, 2004-2013. 
para el 2010. En tanto, el tratamiento sin aplicación de fósforo al voleo se mantuvo con un rendimiento relativo por debajo del $70 \%$. La Figura 8B muestra el comportamiento de los tratamientos sin aplicación de P en banda a partir del año 2010 hasta el 2013; en este periodo todos los tratamientos, con excepción del testigo, tendieron a disminuir el rendimiento relativo. Los tratamientos a base de 50 y $75 \mathrm{~kg} / \mathrm{ha}$ presentaron un rendimiento relativo alrededor del $50 \%$ para el año 2013, mientras que a base de 0 y $25 \mathrm{~kg} /$ ha estuvieron por debajo del $30 \%$. El tratamiento a base de $100 \mathrm{~kg} /$ ha fue el de menor reducción con una media alrededor del $80 \%$ en el 2013. Esta respuesta se explica por la reducida movilidad del ión ortofosfato y la retención (fijación, adsorción e inmovilización) del fertilizante fosfatado en el suelo que requiere de la aplicación localizada del mismo, especialmente en suelos de bajo contenido de $\mathrm{P}$ disponible y en siembras tempranas (Randall y Hoeft, 1988).

Estos resultados sugieren que el efecto residual de la aplicación del fósforo al voleo e incorporado previo a la siembra, varía según la dosis empleada inicialmente, en donde las dosis superiores a los $50 \mathrm{~kg} /$ ha deben repetirse después de siete años para mantener un alto porcentaje de productividad, ya que a partir de este tiempo se reduce drásticamente el efecto residual.

\section{Interacción voleo $\mathrm{x}$ banda $\mathrm{x}$ año}

En la Figura 9 se observa la interacción de las aplicaciones al voleo y banda a través de los años. La Figura 9A muestra la respuesta del rendimiento relativo de grano en la franja aplicada con $100 \mathrm{~kg} /$ ha de $\mathrm{P}$ al voleo; en esta franja las distintas dosis en banda se diferenciaron poco los primeros cinco años y luego a partir del sexto año el tratamiento con 0 $\mathrm{kg} / \mathrm{ha}$ comenzó a disminuir en rendimiento. Por otra parte, el comportamiento de las aplicaciones en banda para las fajas de voleo de 75 y $50 \mathrm{~kg} / \mathrm{ha}$ (Figura 9B y 9 C) tuvieron una respuesta similar. En estas fajas el tratamiento en banda a base de 30 y $20 \mathrm{~kg} / \mathrm{ha}$ se mantuvieron con un rendimiento relativo cercano al 90\% durante todo el período del estudio, mientras que los tratamientos a base de 10 y $0 \mathrm{~kg} / \mathrm{ha}$ decrecieron por debajo del 80\% del rendimiento relativo para el 2013. Por otro lado, la franja que no recibió aplicación de fósforo al inicio del estudio (Figura $9 \mathrm{E}$ ), presentó una diferencia entre las cuatro dosis aplicadas en banda desde el inicio del estudio, y todos los tratamientos, con excepción de la dosis de $30 \mathrm{~kg} / \mathrm{ha}$, se redujeron con el pasar de los años. Esta respuesta sugiere que la dosis de $30 \mathrm{~kg} / \mathrm{ha}$, provee las necesidades de fósforo requeridas por el cultivo, por lo cual a través de los años mantuvo su rendimiento relativo por encima del $95 \%$, por el contrario, con la dosis de 20 y $10 \mathrm{~kg} / \mathrm{ha}$, en los primeros años el rendimiento se mantuvo al máximo, debido a que el cultivo obtiene el resto del fósforo de la aplicación inicial al voleo. Al pasar los años el aporte de $\mathrm{P}$ de la aplicación al voleo se reduce debido a la extracción por parte de las plantas en los años anteriores, en consecuencia el rendimiento de grano de los últimos años se reduce.

\section{Análisis económico}

El análisis económico de presupuesto parcial se realizó a la suma de los diez años, donde se observó que el tratamiento con la aplicación de $75 \mathrm{~kg} \mathrm{P} / \mathrm{ha}$ al voleo el primer año y sin aplicación en banda por año, presentó una tasa de retorno marginal (TRM) de $1788 \%$ y resultó el de mayor retorno (Figura 10). Entre todos los tratamientos sobresalió el que consistió de la aplicación al voleo de $100 \mathrm{~kg}$ el primer año más $10 \mathrm{~kg} \mathrm{P} / \mathrm{ha}$ anualmente, con un $91 \%$ de rendimiento relativo. Se debe señalar que el testigo a pesar de tener un buen rendimiento relativo fue superado desde el punto de vista económico por los tratamientos de 25 , 75 y $100 \mathrm{~kg} \mathrm{P} / \mathrm{ha}$ al voleo sin aplicaciones posteriores en bandas. De manera general, los tratamientos al voleo dominaron tanto física como económicamente a la práctica del agricultor consistente en aplicar anualmente la dosis de $30 \mathrm{~kg} \mathrm{P} / \mathrm{ha}$ en forma de banda. Las aplicaciones al voleo de más de $75 \mathrm{~kg}$ de $\mathrm{P} / \mathrm{ha}$ empezaron a decrecer significativamente después de los cinco años de su aplicación. 

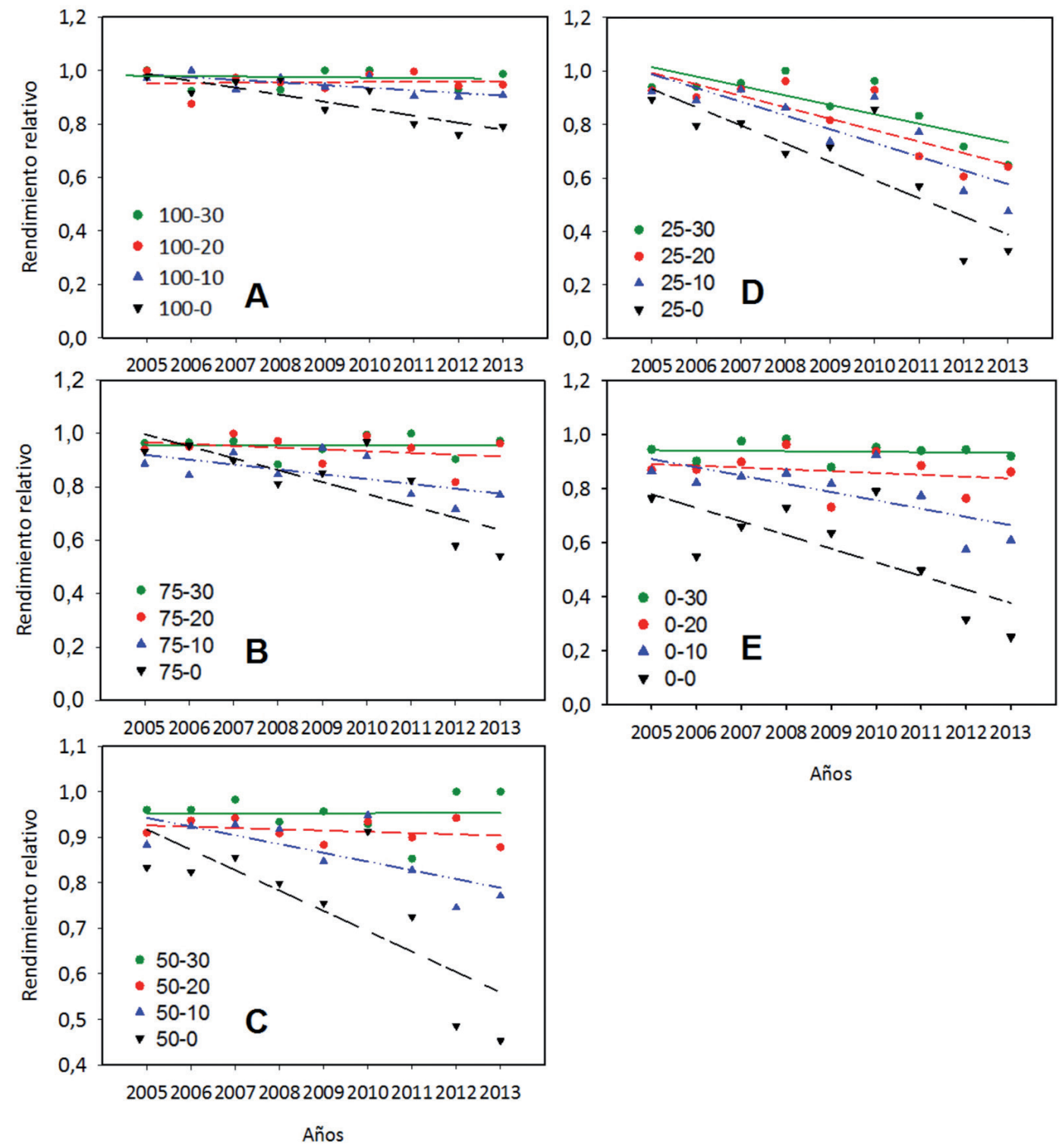

200520062007200820092010201120122013

Años

Figura 9. Rendimiento relativo del grano de maíz en respuesta a la aplicación de distintos niveles de fósforo en banda según dosis de aplicación al voleo de 100 (A), 75 (B), 50 (C), 25 (D) y 0 (E) kg P/ha a través de los años. El Ejido, Panamá. 2004-2013.

Figure 9. Relative grain yield response of the different doses of phosphorus in band application according broadcast doses of 100 (A), 75 (B), 50 (C), 25 (D), and 0 (E) kg P/ha through years. El Ejido, Panama. 2004-2013. 


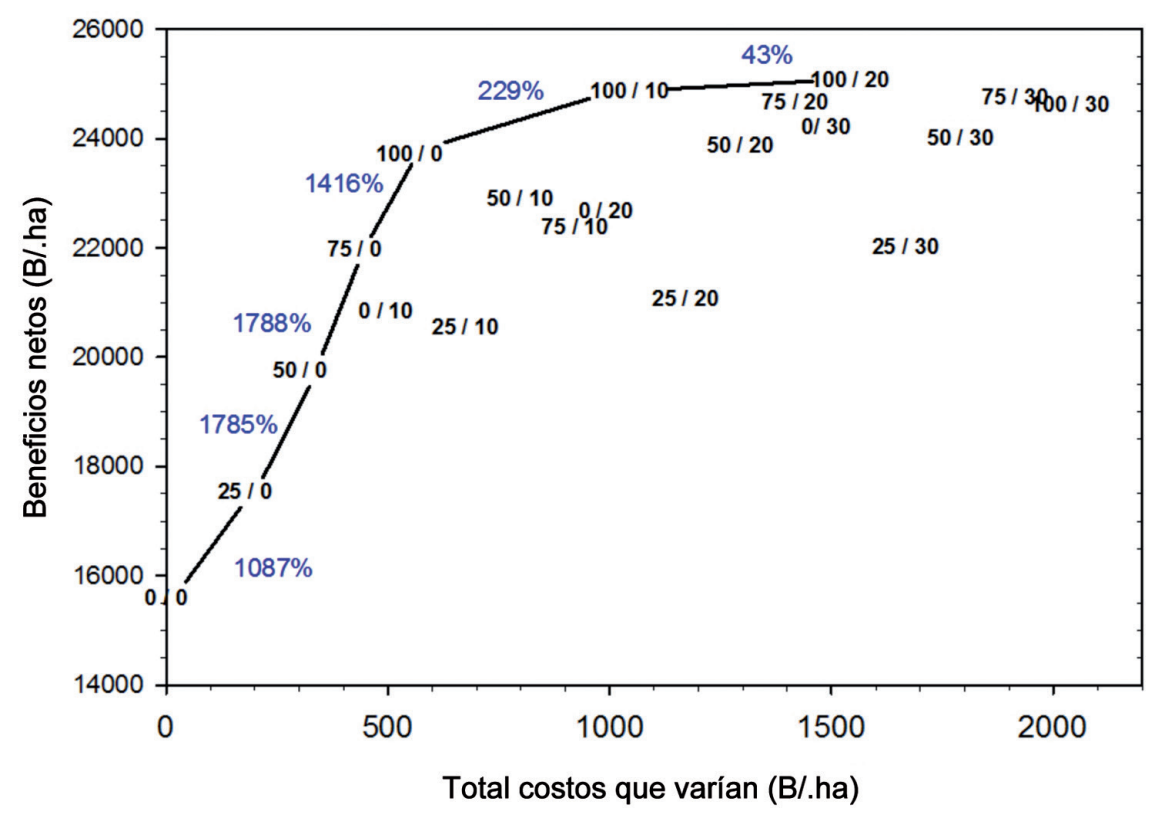

Figura 10. Curva de beneficios netos del ensayo de manejo de la fertilización fosforada en el cultivo de maíz. El Ejido, Panamá. 2004-2013.

Figure 10. Net benefit curve of phosphorus fertilization management in corn crop trial. El Ejido, Panama. 2004-2013.

\section{LITERATURA CITADA}

Bernal, J.H., G.E. Navas, y R.S Hernández. 2014. Requerimientos y respuestas a la fertilización del maíz en suelos de sabanas ácidas de Colombia. IPNI, Inf. Agron. Hispanoamérica 15:6-10.

Bolaños, J., y H. Barreto. 1991. Análisis de los componentes de rendimiento de los ensayos regionales de maíz de 1990. En: W. Raun, H. Barreto, y G. Saín, editores, Análisis de los resultados experimentales del PRM 1990, Vol. 2. CIMMYT, Guatemala, GUA. p 9-27.

Bordoli, J.M., and A.P. Mallarino. 1998. Deep and shallow banding phosphorus and potassium as alternatives to broadcast fertilization for no-till corn. Agron. J. 90:27-33. doi: 10.2134/agronj1998. 00021962009000010006x

Colpo-Gatiboni, L., C.M. Boiera de Oliveira, P.R. Ernani, J.A. Almeida, and D.J. Miquelluti. 2010. Soil available phosphorus by Mehlich-1, Mehlich-3 and anion exchange resin and its sensitivity to soil clay content. [DVD]. 19th World Congress of Soil Science,
Soil Solutions for a Changing World. 1-6 Aug. 2010, Brisbane, AUS. http://www.iuss.org/19th\%20WCSS/ Symposium/pdf/0827.pdf (accessed 17 apr. 2015).

Cordell, D., J.O. Drangert, and S. White. 2009. The story of phosphorus: global food security and food for thought. Global Env. Change 19:292-305.

Díaz-Romeu, R., y A. Hunter. 1982. Metodología de muestreo de suelos, análisis químico de suelos y tejido vegetal y de investigaciones en invernadero. CATIE, Turrialba, CRC.

Dawson, C.J., and J. Hilton. 2011. Fertilizer availability in a resource-limited world: production and recycling of nitrogen and phosphorus. Food Policy 36:14-22.

Ferraris, G. 2008. Fertilización fosforada en cultivos extensivos. Criterios de diagnóstico y herramientas tecnológicas para su manejo en el norte - Centro-oeste de Buenos Aires y sur de Santa Fe. En: AIANBA, editores. IV Jornada de Maíz INTA EEA Pergamino. AIANBA 23 de julio de 2008, Pergamino, ARG. 9 p.

García, F. 2001. Fertilización del maíz en la región pampeana. En: AIANBA, editores, Actas VII Congreso Nacional 
de Maíz. AIANBA 7 al 9 noviembre de 2001. Pergamino, Buenos Aires, ARG. 6 p.

Gordon, R., N. De Gracia, A. González, J. Franco, y A.P. de Herrera. 1995. Respuesta del cultivo de maíz a la aplicación de Fósforo y Azufre, Panamá 1989-1992. Rev. Cienc. Agropecu. 8:193-214.

Gordon, R., N. De Gracia, A. González, J. Franco, A. Herrera, y S. Jaramillo. 1992. Evaluación de la respuesta física y económica al $\mathrm{N}, \mathrm{P}$ y $\mathrm{K}$ en el cultivo de maíz en la Región de Azuero, Panamá, 1991. En: IDIAP, editores. Síntesis de los Resultados Experimentales del Programa de Maíz Panamá 19911992. IDIAP, Los Santos, PAN. p. 135-141.

Gordon, R., J. Franco, y A. González. 2004. Determinación de la dosis óptima de Nitrógeno para el cultivo de maíz con tres modelos de respuesta, Azuero, Panamá, 2000-2002. Rev. Cienc. Agropecu. 15:1-16.

Gordon, R., A. González, y J. Franco. 2000. Manejo de la fertilización nitrogenada en el cultivo de maíz. Azuero, Panamá, 1996-1998. Rev. Cienc. Agropecu. 10:123-134.

Hinsinger, P., E. Betencourt, L. Bernard, A. Brauman, C. Plassard, J. Shen, X. Tang, and F. Zhang. 2011. P for two, sharing a scarce resource e soil phosphorus acquisition in the rhizosphere of intercropped species. Plant Physiol. 156:1078-1086.

Howard, D.D., M.E. Essington, and J. Logan. 2002. Longterm broadcast and banded phosphorus fertilization of corn produced using two tillage systems. Agron. J. 94:51-56. doi: 10.2134/ agronj2002.5100

Jauregui, M.A., and G. Saín. 1990. Continuous economic analysis of crop response to fertilizer in on farm research. CIMMYT, México D.F., MEX.

Khan, S., and M. Hanjra. 2009. Footprints of water and energy inputs in food production - Global perspectives. Food Policy 34:130-140.

Littell, R.C., G.A. Milliken, W.W. Stroup, R.D. Wolfinger, and O. Schabenberger. 2006. SAS for mixed models. 2nd. ed. SAS Institute Inc. Cary, NC, USA.

McWilliams, D.A., D.R. Berglund, and G.J. Endres. 1999. Corn growth and management quick guide. NDSU Extension Service. University of North Dakota y University of Minnesota. http://library.ndsu.edu/ tools $/ \mathrm{dspace} /$ load $/$ ?file=/repository $/$ bitstream $/$ handle/10365/9112/A1173_1999.pdf?sequence=1 (accessed 17 apr. 2015).

Name, B., y A. Cordero. 1987. Recomendaciones para la fertilización de suelos: Hojas guías por cultivo. En:
S. Jaramillo editor, Compendio de los resultados de Investigación presentados en la Jornada Científica. IDIAP. Santiago, PAN. p. 22.

Pérez, M.J. 1995. Comparación de cuatro métodos extractantes de fósforo disponible en suelos tratados con fuentes de fósforo de diferentes grados de solubilidad. Agron. Trop. 45:507-526.

Pineda-Mares, P., J.F. Martínez, A. Amante, y V.M. Ruíz. 2001. Respuesta del maíz al fósforo y un mejorador de suelos en áreas yesosas de la zona media de San Luís Potosí. Rev. Chapingo, Serie Zonas Áridas 2:106-113.

Prystupa, P., F.H. Gutiérrez, F. Salvagiotti, G. Ferraris, and L. Couretot. 2006. Measuring corn response to fertilization in the Northern Pampas. Better Crops 90 (2):25-27.

Ramírez, R., M.D. Beg, O. Colmenares, E. Meléndez, P. Marbal, F. Blanco, E. Guzmán, C. Hernández, y A. Chirinos. 1988. Relación de la respuesta del maíz a la aplicación de fósforo y el $\mathrm{P}$ asimilable del suelo. Agron. Trop. 38:5-20.

Randall, G., and R. Hoeft. 1988. Placement methods for improved efficiency of $\mathrm{P}$ and $\mathrm{K}$ fertilizers: A review. J. Prod. Agric. 1:70-79. doi:10.2134/jpa1988.0070

Senigagliesi, C., R. García, y M.L. de Galetto. 1984. Evaluación de la respuesta del maíz a la fertilización nitrogenada y fosfatada en el área centro-norte de Buenos Aires y sur de Santa Fe. En: Actas III Congreso Nacional de Maíz. 6 al 9 Nov. 1984. Pergamino, Buenos Aires, ARG.

Villarreal,J.,L. Barahona,y O. Castillo. 2015.Determinación de las principales formas de fósforo en suelos de Panamá. En: Actas LX Reunión Anual del Programa Cooperativo Centroamericano para el Mejoramiento de Cultivos y Animales (PCCMCA), 4 al 7 de mayo de 2015. Ciudad de Guatemala, GUA.

Villarreal, J., B. Name, y R. García. 2013. Zonificación de suelos de Panamá en base a niveles de nutrientes. Rev. Cienc. Agropecu. 21:71-89.

Welch, L.F., D.L. Mulvaney, L.V. Boone, G.E. McKibben, and J.W. Pendleton. 1966. Relative efficiency of broadcast versus banded phosphorus for corn. Agon. J. 58:283:287. doi:10.2134/agronj1966.00021962005 $800030011 \mathrm{x}$ 